DOI: http://dx.doi.org/10.18524/1810-4215.2017.30.114351

\title{
H $\alpha$ AND H $\beta$ PROFILE VARIATIONS IN THE SPECTRA OF THE STAR 55 CYG
}

\author{
Y.M. Maharramov \\ Shamakhy Astrophysical Observatory, Azerbaijan National Academy of \\ Sciences, Yu. Mammadaliyev settlement, Shamakhy district, Republic of Azerbaijan \\ y_meherremov@rambler.ru
}

ABSTRACT. We study the variability of the $\mathrm{H} \alpha$ and $\mathrm{H} \beta$ lines in the spectra of $55 \mathrm{Cyg}$ based on observations acquired in 2014 on the 2-m telescope of Shamakhy Astrophysical Observatory. In this work line profiles of $\mathrm{H} \alpha$ and $\mathrm{H} \beta$ are described extensively and compared with early observations. The profile of $\mathrm{H} \alpha$ line vanishes or almost vanishes in the spectra on some epochs again. Our observations showed that when the $\mathrm{H} \alpha$ line disappeared or weaken sharply in the spectra, the HeI and $\mathrm{H} \beta$ line are redshifted, but no synchronous variabilities were observed in other spectral lines (CII and others) formed in deeper layers of the stellar atmosphere. In addition, the profiles of the $\mathrm{H} \alpha$ and $\mathrm{H} \beta$ lines have been analysed, as well as their relations with possible conditions of the atmosphere of 55 Cyg.

We revealed that the structures of photospheric (CII, HeI etc.) absorption line profiles, as well as $\mathrm{H} \beta$ line, their radial velocities and equivalent widths are variable.

These events may be associated with a manifestation of the non-stationarity of the atmosphere or non-sphericity of the stellar wind.

Keywords: Supergiant star, stellar atmosphere, P Cyg profile, $\mathrm{H} \alpha$ and $\mathrm{H} \beta$ line profiles.

\section{Introduction}

HD198478 (55 Cyg) is a hot supergiant of spectral type B3Ia with the following parameters (Wilson, 1953; Barlow \& Cohen, 1977; Gies \& Lambert, 1992; Crowther et al., 2006; Markova \& Puls, 2008):

$$
\begin{gathered}
m_{\mathrm{v}}=4.86 \mathrm{mag}, T_{\text {eff }}=17500 \mathrm{~K}, M / M_{\odot}=11, R / R_{\odot}=49, \log \\
L / L_{\odot}=5.31, \log g=2.10, V_{\mathrm{rad}}=-7.2 \mathrm{~km} / \mathrm{s}, \xi=20 \mathrm{~km} / \mathrm{s} \\
v \operatorname{sini}=61 \mathrm{~km} / \mathrm{s}, d=0.97 \mathrm{kpc}
\end{gathered}
$$

It is believed that this star belongs to Cyg OB7 association (Crowther et al., 2006).

Studying of the photospheric lines of $55 \mathrm{Cyg}$ Hutchings (1970) revealed interesting facts: different elements typically different radial velocities, while the same lines show strong variations with time. In addition, Underhill (1960) described radial velocity variations of 30 $\mathrm{km} / \mathrm{s}$, but in these changes no found periodicity.
Later, Granes (1975) collected 34 spectra, based on spectral observations of the star over 15 consecutive nights, and found that the radial velocity curves oscillate with a period of four-to-five days, in addition to photospheric motions on timescales about three times longer. Kraus et al. (2015) studied the interplay between pulsations and mass loss in the blue supergiant 55 Cyg. They modeled the H, HeI, SiII, and SiIII lines using the non-local thermal equilibrium atmosphere code FASTWIND and derived the photospheric and wind parameters. In addition, they searched for variability in the intensity and radial velocity of photospheric lines and performed a moment analysis of the line profiles to derive frequencies and amplitudes of the variations. The $\mathrm{H} \alpha$ line varies with time in both intensity and shape, displaying various types of profiles: $P$ Cyg, pure emission, almost complete absence, and double or multiple peaked. They also observed changes in the ionization rate of SiII and determined the multiperiodic oscillation in the HeI absorption lines, with periods ranging from a few hours to 22.5 days, and interpreted the photospheric line variations in terms of oscillations in $\mathrm{p}$-, g-, and strange modes. Authors suggested that these pulsations can lead to phases of enhanced mass loss.

The profile of $\mathrm{H} \alpha$ line in the spectra of $55 \mathrm{Cyg}$ is also highly variable.

Underhill (1960) remarked that in her spectra the $\mathrm{H} \alpha$ line maintained a P Cyg profile, but with highly variable strength. Ebbets (1982) noted that during one of his observations, $\mathrm{H} \alpha$ line showed additional faint emission wings, which were not visible anymore in the spectrum.

As seen, researchers noted that, $\mathrm{H} \alpha$ line in $55 \mathrm{Cyg}$ indicates fast variable structure, but the sequence of observations was irregular and inadequate to trace in detail the changes in the spectra. Therefore they noted that more and systematic observations are needed to investigate this supergiant.

In the present paper we analyzed variations of the $\mathrm{H} \alpha$ and $\mathrm{H} \beta$ lines.

We also investigated the variabilities of the CII (6578.05 $\AA, 6582.88 \AA$ ), and HeI (5875.72 $\AA$ ) lines which formed deeper effective layers in the atmosphere of this star. Our main aim is to study the observed peculiarities of these lines in the spectra. 

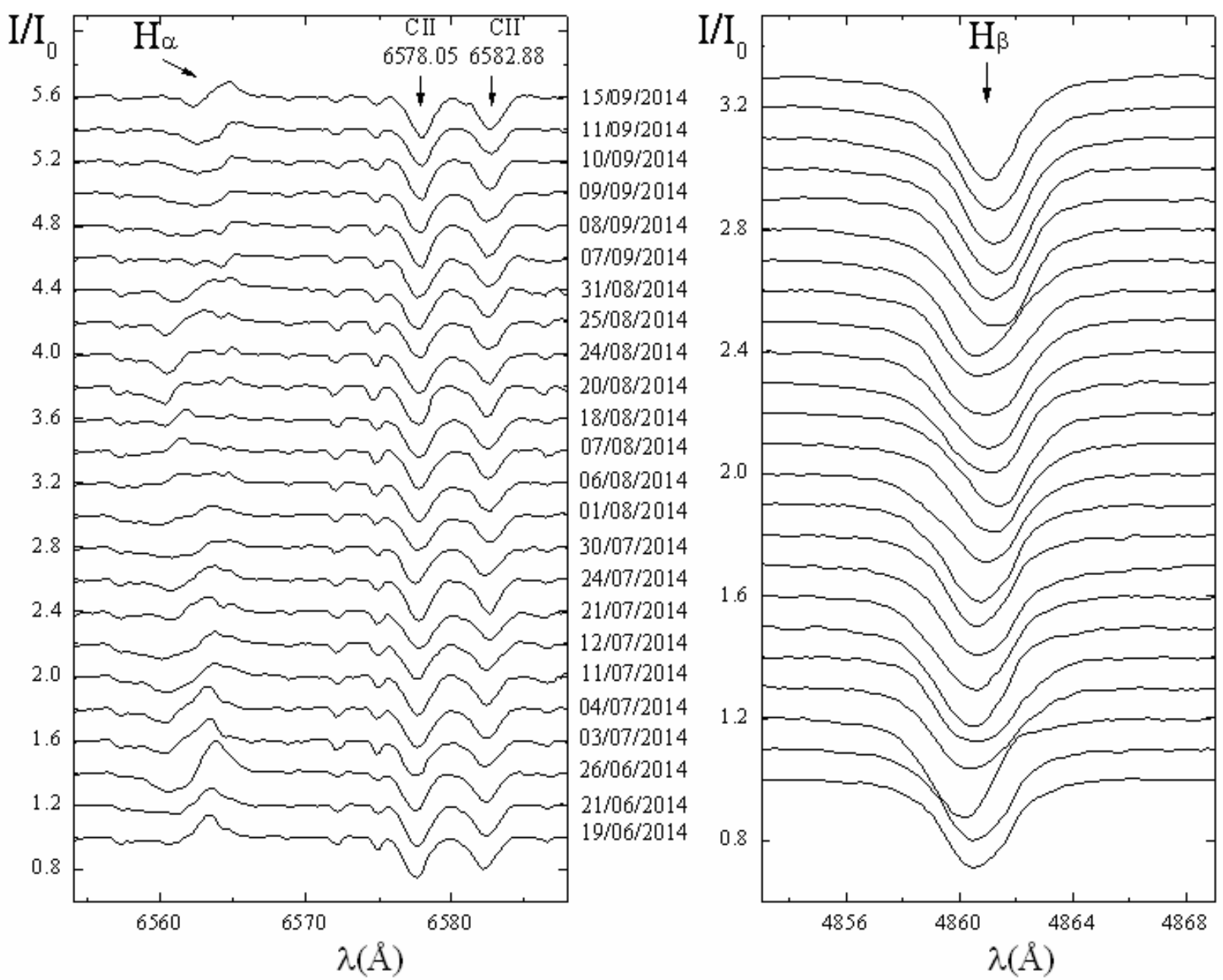

Figure 1: Profiles of the H $\alpha, \mathrm{CII}$ and $\mathrm{H} \beta$ lines in the spectra of 55 Cyg observed in 2014.

\section{Observations and Data processing}

Spectral observations of 55 Cyg were acquired during from June 19, 2014 to September 15, 2014 using a CCD detector in the echelle spectrometer mounted at the Cassegrain focus of the 2-m telescope of the Shamakhy Astrophysical Observatory (Mikailov et al., 2005). The spectral resolution was $\mathrm{R}=15000$ and the spectral range is $\lambda \lambda 4700-6700 \AA \AA$. One to two spectra of the target star were obtained on each night. The signal-to-noise ratio was $\mathrm{S} / \mathrm{N}=150-200$. The average exposure was $600-900 \mathrm{~s}$, depending on the weather conditions.

The Echelle spectra were processed with the standard technique using the DECH20 software package (Galazutdinov, 1992). The reduction of the spectra, which included the continuum placement, the construction of dispersion curves (from the spectra of a hollow-cathode $\mathrm{Th}+\mathrm{Ar}$ lamp or radial velocity standard stars), spectrophotometric and position measurements was performed using this package.

In addition to the observations of the target star, in order to check the stability of the instrument we also obtained numerous spectra of standard stars, the daily sky solar spectrum, and comparison spectra. Thus, we recorded the spectrum of a thorium-argon lamp for wavelength calibration. The stability of the wavelength scale was verified by measuring the wavelength centroids of $\mathrm{OI}$ and $\mathrm{H}_{2} \mathrm{O}$ sky lines. The long term accuracy achieved for the wavelength calibration is of the order of $1 \mathrm{~km} / \mathrm{s}$ as derived from the spread of measured radial velocities of telluric lines in the spectra. The measurement error for the equivalent widths (W) does not exceed $5 \%$, and error of the radial velocity $(\mathrm{Vr})$ is of the order of $\pm 2 \mathrm{~km} / \mathrm{s}$. Here, $(\mathrm{Vr})$ are velocities of the absorption and emission peaks in the selected lines. Note that appropriate heliocentric corrections were included during data processing.

\section{Results}

The $\mathrm{H} \alpha$ line varies with time in both intensity and shape, displaying various types of profiles: normal P Cyg, pure absorption, pure emission, double or triple-peaked emission and complete absence.

We found that the complete absence of $\mathrm{H} \alpha$ line profile is real and recurrent. The $\mathrm{H} \beta$ line profiles and the radial velocities change during the observation period. When the $\mathrm{H} \alpha$ line disappears or weaken sharply the HeI and $\mathrm{H} \beta$ lines are redshifted.

When the $\mathrm{H} \alpha$ line disappeared in the spectra, no significant variabilities were observed in other spectral lines (except $\mathrm{HeI}$ and $\mathrm{H} \beta$ ) formed in deeper layers of the stellar atmosphere. 


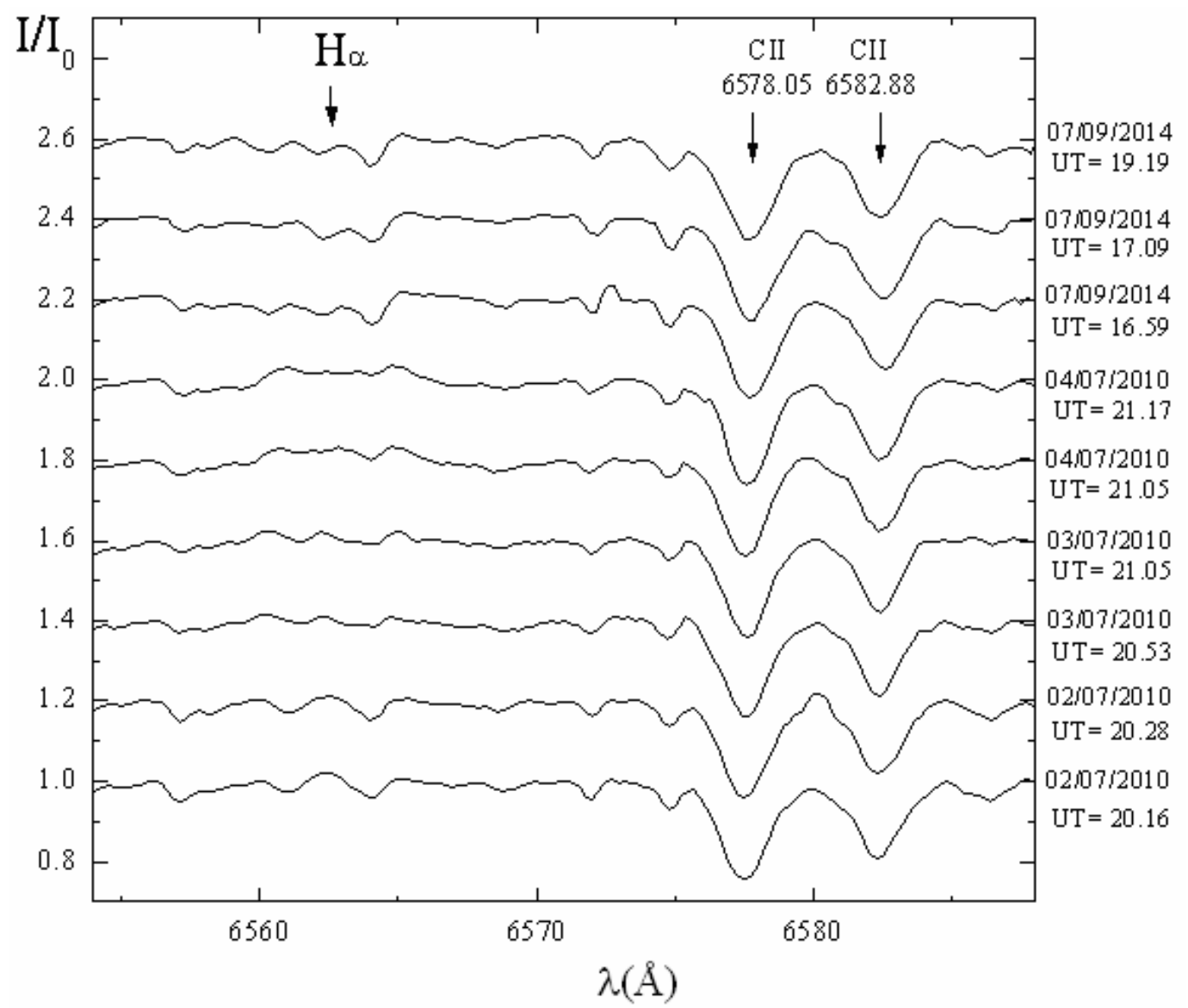

Figure 2: H $\alpha$ and CII profiles in the individual spectra of 55 Cyg observed in 2010 and 2014.

In addition, in several spectra the emission components of $\mathrm{H} \alpha$ line are observed to be double or triple (Fig. 1).

During the observational period hydrogen and photospheric absorption line profiles and their radial velocities exhibited variations. The radial velocities measured from the absorption line cores vary with time and with the line intensity.

\section{Data Analysis}

It is known that the $\mathrm{H} \alpha$ line in the spectra of $\mathrm{B}$ supergiants has a clear P Cyg-type profile.

Our observations show that the most pronounced variability in the spectra of $55 \mathrm{Cyg}$ are noticeable in the intensity and profiles of the $\mathrm{H} \alpha$ line. The following shapes of the $\mathrm{H} \alpha$ profile were observed: normal P Cyg profile; normal $\mathrm{P}$ Cyg-type profile with double-peaked in emission; normal P Cyg-type profile with triple-peaked in emission; pure emission profile; pure absorption profile; complete absence.

Fig. 1 shows the spectra of $55 \mathrm{Cyg}$ in the wavelength ranges covering the $\mathrm{H} \alpha$ and $\mathrm{H} \beta$ regions observed in 2014. Here, the spectra obtained on 20/08/2014, and 09/09/2014 are shown individual profiles, only on 07/09/2014 are averaged three expositions, but the rest of them are averaged two expositions taken during single nights. The spectra are shifted vertically by an amount that ensures better visibility. On the right of $\mathrm{H} \alpha$ the profiles of the CII resonance doublet are seen. Vertical lines represent the laboratory position of the lines.
$\mathrm{H} \alpha$ shows variations in strength in both the absorption and emission components of the P Cyg profile (see Fig.1). The pattern of variability in 2014 can be described in the following qualitative way:

P Cyg profile with weak absorption, slowly evolves into P Cyg with deep components, later both components are weaken relatively, absorption components are broader and emissions is observed a double-peaked, only emission with triple-peaked is seen, later weaker absorption and emission components with triple-peaked are observed, both components (emission with double-peaked) are deeper, on September 07, 2014, the H $\alpha$ disappeared, and on September 08, 2014, the $\mathrm{H} \alpha$ line became faint, on September 09, 2014, the absorption component became stronger and later emission component also became stronger, at last on September 15, 2014, normal P Cyg profile of $\mathrm{H} \alpha$ is observed again. As can be seen here more interesting event is the disappearance of $\mathrm{H} \alpha$ profile from the spectra. All spectral measurements presented in Table 1.

Note that similar patterns were observed in 2010 (Maharramov, 2013). So, the first time the $\mathrm{H} \alpha$ line alone is absent from the spectra on July 02-04, 2010, and no spectral features are observed at this wavelength (6562.816 $\AA$ ), except for weak atmospheric lines and noise. In those spectra, all the other lines typical for hot supergiants such as $55 \mathrm{Cyg}$, apart from $\mathrm{H} \alpha$, are observed, including $H \beta$. In addition, at those times we revealed that the $\mathrm{H} \beta$ equivalent width increased and a shift of this line toward the red is observed when $\mathrm{H} \alpha$ is absent. 
Table 1. Values of the radial velocity (Vr), equivalent width (W), and depth (R) or residual intensity (r) in the H $\alpha$ line.

\begin{tabular}{|c|c|c|c|c|c|c|c|}
\hline Date & JD 2450000+ & $\begin{array}{c}\operatorname{Vr}(\mathrm{abs}) \\
\mathrm{km} / \mathrm{s}\end{array}$ & $\begin{array}{c}\mathrm{Vr}(\mathrm{em}) \\
\mathrm{km} / \mathrm{s}\end{array}$ & $\begin{array}{c}\mathrm{W}(\mathrm{abs}) \\
\AA\end{array}$ & $\begin{array}{c}\mathrm{W}(\mathrm{em}) \\
\AA\end{array}$ & $\mathrm{R}(\mathrm{abs})$ & $\mathrm{r}(\mathrm{em})$ \\
\hline $\begin{array}{c}2014 \\
\text { Jun.19 }\end{array}$ & 6828.40 & -83 & 43 & 0.15 & 0.25 & 0.04 & 1.14 \\
\hline Jun.21 & 6830.41 & -73 & 47 & 0.20 & 0.19 & 0.06 & 1.10 \\
\hline Jun.26 & 6835.34 & -92 & 55 & 0.36 & 0.41 & 0.11 & 1.20 \\
\hline Jul.03 & 6842.29 & -105 & 39 & 0.22 & 0.23 & 0.08 & 1.13 \\
\hline Jul.04 & 6843.29 & -104 & 33 & 0.24 & 0.24 & 0.09 & 1.13 \\
\hline Jul.11 & 6850.32 & -93 & $\begin{array}{l}50 \\
98\end{array}$ & 0.34 & 0.18 & 0.10 & $\begin{array}{l}1.08 \\
1.06\end{array}$ \\
\hline Jul.12 & 6851.31 & -113 & $\begin{array}{l}50 \\
98\end{array}$ & 0.38 & 0.14 & 0.10 & $\begin{array}{l}1.07 \\
1.05\end{array}$ \\
\hline Jul.21 & 6860.31 & -88 & $\begin{array}{c}28 \\
101\end{array}$ & 0.16 & 0.21 & 0.05 & $\begin{array}{l}1.09 \\
1.05\end{array}$ \\
\hline Jul.24 & 6863.26 & -88 & $\begin{array}{l}48 \\
89\end{array}$ & 0.21 & 0.18 & 0.07 & $\begin{array}{l}1.08 \\
1.06\end{array}$ \\
\hline Jul.30 & 6869.27 & -88 & $\begin{array}{l}47 \\
96\end{array}$ & 0.27 & 0.08 & 0.06 & $\begin{array}{l}1.04 \\
1.05\end{array}$ \\
\hline Aug.01 & 6871.29 & -118 & $\begin{array}{l}42 \\
91 \\
\end{array}$ & 0.22 & 0.11 & 0.06 & $\begin{array}{l}1.06 \\
1.04\end{array}$ \\
\hline Aug.06 & 6876.33 & - & $\begin{array}{c}-54 \\
42 \\
88 \\
\end{array}$ & - & 0.25 & - & $\begin{array}{l}1.06 \\
1.06 \\
1.06 \\
\end{array}$ \\
\hline Aug.07 & 6877.31 & - & $\begin{array}{c}-53 \\
7 \\
\end{array}$ & - & 0.17 & - & $\begin{array}{l}1.08 \\
1.05 \\
\end{array}$ \\
\hline Aug.18 & 6888.31 & -113 & $\begin{array}{c}-41 \\
40 \\
91\end{array}$ & 0.07 & 0.08 & 0.05 & $\begin{array}{l}1.05 \\
1.01 \\
1.02\end{array}$ \\
\hline Aug.20 & 6890.31 & -118 & $\begin{array}{c}-49 \\
33 \\
89 \\
\end{array}$ & 0.24 & 0.12 & 0.11 & $\begin{array}{l}1.03 \\
1.03 \\
1.06 \\
\end{array}$ \\
\hline Aug.24 & 6894.21 & -105 & $\begin{array}{l}35 \\
93\end{array}$ & 0.31 & 0.06 & 0.12 & $\begin{array}{l}1.02 \\
1.04\end{array}$ \\
\hline Aug.25 & 6895.23 & -108 & $\begin{array}{l}11 \\
87\end{array}$ & 0.13 & 0.17 & 0.09 & $\begin{array}{l}1.07 \\
1.05\end{array}$ \\
\hline Aug.31 & 6901.22 & -89 & $\begin{array}{l}40 \\
88 \\
\end{array}$ & 0.15 & 0.20 & 0.07 & $\begin{array}{l}1.06 \\
1.07 \\
\end{array}$ \\
\hline Sep.07 & 6908.21 & - & - & - & - & - & - \\
\hline Sep.08 & 6909.26 & $?$ & $?$ & $?$ & $?$ & $?$ & $?$ \\
\hline Sep.09 & 6910.16 & $\begin{array}{c}-21 \\
20 \\
\end{array}$ & 106 & 0.37 & 0.02 & $\begin{array}{l}0.08 \\
0.07 \\
\end{array}$ & 1.01 \\
\hline Sep.10 & 6911.16 & -15 & 93 & 0.23 & 0.03 & 0.08 & 1.02 \\
\hline Sep.11 & 6912.13 & -19 & 92 & 0.27 & 0.06 & 0.09 & 1.04 \\
\hline Sep. 15 & 6916.26 & -28 & 84 & 0.09 & 0.15 & 0.06 & 1.09 \\
\hline
\end{tabular}

Furthermore, Kraus et al. (2015) discovered that $\mathrm{H} \alpha$ profile almost completely disappears from the spectrum again, and in their data they recognized it in 2012, July, 24 and in 2013, July, 26. Authors speculated that the wind conditions of $55 \mathrm{Cyg}$ may have changed, because no earlier observational work on this star mentions phases in which the wind emission swamps the photospheric absorption line.

We noted above that on September 07, 2014, the H $\alpha$ profile is absent from the spectrum of $55 \mathrm{Cyg}$ again. Therefore, we suggest that this behavior is recurrent process.
Unfortunately, weather conditions before July 02-04, 2010 and September 07, 2014, did not make it possible to obtain spectra of the star $55 \mathrm{Cyg}$, which could shed light on the transition moments of this interesting process.

In order to investigate the short term variability in the $\mathrm{H} \alpha$ profile, all individual spectra obtained on July 02-04, 2010, and on September 07, 2014 were processed separately. These data are presented in Fig. 2. As can be seen the $\mathrm{H} \alpha$ profile can be variable on a time scale of hours.

In Table 1 the radial velocities, equivalent widths and the depths (and residual intensities) of absorption and emission components of $\mathrm{H} \alpha$ are presented. Our measurements show 
Table 2. Measurements of the radial velocity, equivalent width and depth of the H $\beta$, CII, and HeI lines.

\begin{tabular}{|c|c|c|c|c|c|c|c|c|c|c|}
\hline \multirow{2}{*}{ Date } & \multirow{2}{*}{ JD 2450000+ } & \multicolumn{2}{|c|}{ CII6578.05尺 } & \multicolumn{2}{|c|}{ CII6582.88§ } & \multicolumn{2}{|c|}{ HeI5875.72 } & \multicolumn{3}{|c|}{$\mathrm{H} \beta$} \\
\hline & & $\begin{array}{c}\mathrm{Vr} \\
\mathrm{km} / \mathrm{s}\end{array}$ & $\begin{array}{l}\mathrm{W} \\
\AA\end{array}$ & $\begin{array}{c}\mathrm{Vr} \\
\mathrm{km} / \mathrm{s}\end{array}$ & $\begin{array}{l}\mathrm{W} \\
\AA \\
\end{array}$ & $\begin{array}{c}\mathrm{Vr} \\
\mathrm{km} / \mathrm{s}\end{array}$ & $\begin{array}{l}\mathrm{W} \\
\AA\end{array}$ & $\begin{array}{c}\mathrm{Vr} \\
\mathrm{km} / \mathrm{s}\end{array}$ & $\begin{array}{l}\mathrm{W} \\
\AA \\
\end{array}$ & $\mathrm{R}$ \\
\hline $\begin{array}{c}2014 \\
\text { Jun.19 }\end{array}$ & 6828.40 & -9 & 0.50 & -9 & 0.40 & -13 & 0.77 & -38 & 0.93 & 0.29 \\
\hline Jun.21 & 6830.41 & -8 & 0.53 & -11 & 0.40 & -13 & 0.77 & -41 & 1.00 & 0.30 \\
\hline Jun.26 & 6835.34 & -10 & 0.47 & -12 & 0.38 & -20 & 0.76 & -7 & 1.01 & 0.33 \\
\hline Jul.03 & 6842.29 & -9 & 0.47 & -10 & 0.35 & -14 & 0.82 & -47 & 0.99 & 0.26 \\
\hline Jul.04 & 6843.29 & -8 & 0.54 & -9 & 0.41 & -9 & 0.89 & -35 & 1.02 & 0.28 \\
\hline Jul.11 & 6850.32 & -8 & 0.48 & -8 & 0.35 & -15 & 0.84 & -40 & 1.07 & 0.33 \\
\hline Jul.12 & 6851.31 & -9 & 0.46 & -13 & 0.35 & -14 & 0.85 & -34 & 1.06 & 0.31 \\
\hline Jul.21 & 6860.31 & -2 & 0.54 & -4 & 0.41 & -9 & 0.77 & -35 & 0.96 & 0.29 \\
\hline Jul.24 & 6863.26 & -5 & 0.51 & -6 & 0.41 & -12 & 0.78 & -38 & 0.97 & 0.30 \\
\hline Jul.30 & 6869.27 & -15 & 0.47 & -15 & 0.40 & -13 & 0.83 & -30 & 1.07 & 0.32 \\
\hline Aug.01 & 6871.29 & -6 & 0.51 & -8 & 0.37 & -6 & 0.84 & -15 & 1.03 & 0.29 \\
\hline Aug.06 & 6876.33 & -9 & 0.53 & -11 & 0.40 & -5 & 0.77 & 1 & 0.93 & 0.29 \\
\hline Aug.07 & 6877.31 & -9 & 0.48 & -9 & 0.32 & 2 & 0.81 & 9 & 1.01 & 0.31 \\
\hline Aug.18 & 6888.31 & -12 & 0.53 & -15 & 0.41 & -9 & 0.85 & -14 & 1.04 & 0.30 \\
\hline Aug.20 & 6890.31 & -11 & 0.47 & -20 & 0.34 & -12 & 1.00 & -15 & 1.09 & 0.32 \\
\hline Aug.24 & 6894.21 & -9 & 0.52 & -14 & 0.39 & -6 & 0.88 & -26 & 1.09 & 0.30 \\
\hline Aug.25 & 6895.23 & -10 & 0.47 & -12 & 0.35 & -15 & 0.83 & -39 & 1.08 & 0.28 \\
\hline Aug.31 & 6901.22 & -16 & 0.52 & -18 & 0.31 & -24 & 0.83 & -48 & 1.08 & 0.31 \\
\hline Sep.07 & 6908.21 & -17 & 0.53 & $\begin{array}{l}-17 \\
\end{array}$ & 0.37 & -7 & 0.85 & -4 & 1.30 & 0.32 \\
\hline Sep.08 & 6909.26 & -11 & 0.50 & -18 & 0.38 & -10 & 0.89 & -13 & 1.05 & 0.33 \\
\hline Sep.09 & 6910.16 & -14 & 0.53 & -23 & 0.40 & -5 & 0.87 & -14 & 1.16 & 0.34 \\
\hline Sep.10 & 6911.16 & -12 & 0.44 & -12 & 0.30 & -7 & 0.83 & -17 & 1.19 & 0.35 \\
\hline Sep.11 & 6912.13 & -11 & 0.47 & -12 & 0.31 & -8 & 0.84 & -19 & 1.14 & 0.33 \\
\hline Sep.15 & 6916.26 & -8 & 0.51 & -11 & 0.43 & -11 & 0.83 & -21 & 1.09 & 0.34 \\
\hline
\end{tabular}

that the spectral parameters $(\mathrm{W}, \mathrm{R}, \mathrm{r})$ and radial velocities of the absorption and emission components of the H $\alpha$ line, as well as the parameters of lines of other elements, also vary with time (see Table 1 and 2). The largest variations are displayed by the emission component of the $\mathrm{H} \alpha$ line, which indicates changes of the physical condition in the expanding stellar envelope.

As seen from the Table 1 the significant changes happened in the radial velocity and spectral parameters of the $\mathrm{H} \alpha$ line. Fig. 1 , and Table 1 show that sometimes, $\mathrm{H} \alpha$ line absorption component is observed shifted toward the red side with a velocity of $-15 \mathrm{~km} / \mathrm{s}$. Table 1 also shows that the radial velocity of the "blue" component of the absorption line $\mathrm{H} \alpha$ varies within from -118 to $-15 \mathrm{~km} / \mathrm{s}$. The radial velocity of the $\mathrm{H \alpha}$ line blue emission component varies within the range of $-54 \div+106 \mathrm{~km} / \mathrm{s}$. We have determined that the equivalent width, and depth (or the residual intensities in emission) of the absorption

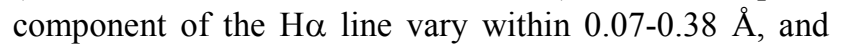
$0.04-0.12$, the emission component varies within 0.02 $0.41 \AA$, and $1.00-1.20$, respectively. Also, we noted above that $\mathrm{H} \alpha$ shows absorption and emission components with double or triple-peaked. If so to speak, the intensity inversions in the emission part of the $\mathrm{H} \alpha$ profile are observed and these are especially interesting.
To investigate the reason for variability of the $\mathrm{H \alpha}$ profile other lines $(\mathrm{H} \beta$, lines of CII and HeI and others) were analyzed in the spectra. As can be seen in Fig. 1, Table 2 when the disappearance of $\mathrm{H} \alpha$ line is observed, the $\mathrm{H} \beta$ line is shifted toward the red simultaneously, and the $H \beta$ equivalent width increases. The $H \beta$ line profile also shows structural changes. Table 2 shows that radial velocity, equivalent width, and depth of the $\mathrm{H} \beta$ line varies within from -48 to $9 \mathrm{~km} / \mathrm{s}, 0.93-1.30 \AA$, and $0.26-0.35$, respectively.

Fig. 1, and Table 2 show that the shape of the CII lines change relatively during observations, as well as the radial velocities of the these lines varied between from -23 to -2 $\mathrm{km} / \mathrm{s}$. The equivalent width varies within $0.44-0.54 \AA$ (for

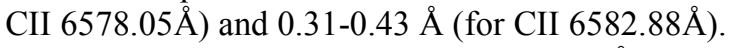

The investigation of the HeI $5875.72 \AA$ line showed that the radial velocity and equivalent width of this line varies between from -24 to $2 \mathrm{~km} / \mathrm{s}$ and $0.76-1.00 \AA$ (Table 2), and its profile also changed relatively (Fig. 3 ). As can be seen in Table 2 when the disappearance of $\mathrm{H} \alpha$ line is observed, the HeI line is also shifted toward the red simultaneously.

We also looked through the NaID doublet lines in the spectra (Fig. 3). Significantly structural changes had not been found in these profiles and the radial velocity varied between $-12 \pm 2 \mathrm{~km} / \mathrm{s}$. 


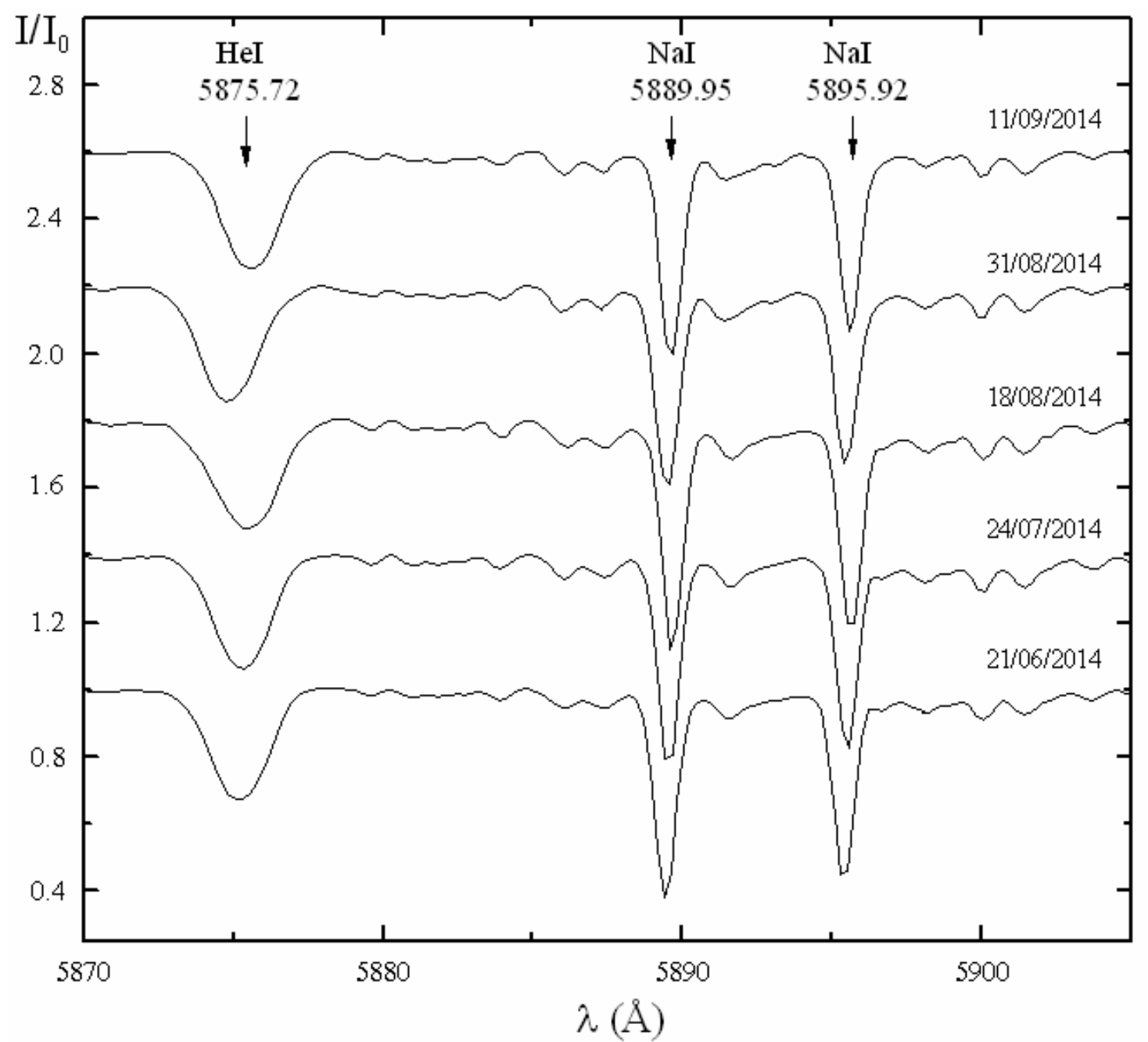

Figure 3: HeI and NaID doublet line profiles of 55 Cyg on different dates

Our measurements show that the line-profile variability (LPV) detected manifests itself by variations in velocity and line-strength, i.e. equivalent width. In particular, we found that the equivalent width, determined by means of line-flux integration within the profiles, varies within \pm 15 $\%$ around a mean value of $0.50 \AA$ (for CII $6578.05 \AA$ ) and $0.35 \AA$ (for CII $6582.88 \AA$ ), as well as, for HeI $5875.72 \AA$ varies within $\pm 15 \%$ around a mean value of $0.83 \AA$. All these indicate the variability of the $\mathrm{H} \beta$, CII and HeI lines.

It has been revealed that the variability of radial velocity and equivalent widths of $\mathrm{H} \beta$ line, as well as the radial velocities of averaged CII and HeI $5875.72 \AA$ lines indicate repeating features.

There appears to be no clear correlation between variations in RVs and change in equivalent width. Fourier analysis performed for the 2014 spectroscopic series did not detect any periodicity for these lines.

In addition, we measured radial velocities of all photospheric absorption lines (CII, HeI, FeII, FeIII, AlIII, SiII, SiIII, NII, NeI, SII etc.) in the spectra and averaged them, and determined for mean velocity $\mathrm{Vr}^{\prime}=-8.5 \mathrm{~km} / \mathrm{s}$.

\section{Discussion}

The profile of the H $\alpha$ line observed in the atmosphere of the supergiant $55 \mathrm{Cyg}$ indicates rapidly variable structure. The dramatic variations previously reported in $55 \mathrm{Cyg}$ (Underhill, 1960; Hutchings, 1970; Granes, 1975) are also seen in our data. Fig. 1 showed that $\mathrm{H} \alpha$ exhibited the normal P Cyg type profile shape in most case. Our spectra enabled us to study the fine structure of the $\mathrm{H} \alpha$ line. The red-shifted absorption seen occasionally in $\mathrm{H} \alpha$, is more likely of wind origin and suggests the presence of matter infall at the base of the wind (Markova et al., 2008). Our observations showed that the $\mathrm{H} \alpha$ line disappeared on September 07, 2014, again (Fig. 1), which is a manifestation of some recurrent processes in the star atmosphere.

The first time a similar variability pattern is revealed in 2010 by us (see Fig. 2). In 2010, we suggested that, when the matter of the stellar wind moves away from the observer, the central frequencies of the emission and absorption components can coincide and cancel each other out, making the Ho line disappear (Maharramov, 2013). $\mathrm{H} \alpha$ line profile completely disappeared from the spectrum in 2014 again. It shows that in particular case the wind emission was reduced so that it compensated by 
coincidence for the absorption of $\mathrm{H} \alpha$ line. These events may be a manifestation of a non-stationary atmosphere of the star or a non-spherical stellar wind (Rosendhal 1973, Sobolev 1947, 1985). Note that, at the time of the disappearance of the $\mathrm{H} \alpha$ line or when it becomes faint (in 2014 September 07-08), the $\mathrm{HeI}$ and $\mathrm{H} \beta$ lines are redshifted, and their radial velocities are $-7 \mathrm{~km} / \mathrm{s}$,

$-10 \mathrm{~km} / \mathrm{s}$ for HeI, $-4 \mathrm{~km} / \mathrm{s}$ and $-13 \mathrm{~km} / \mathrm{s}$ for $\mathrm{H} \beta$, respectively (Table 2 ). These observations may provide an evidence for the fact that in those epochs, the layer of matter where $\mathrm{HeI}$ and $\mathrm{H} \beta$ form move from away the observer and later move to the observer with too lower velocity. That is, if we take into account the velocity of mass center $\left(\mathrm{Vr}^{\prime}=-8.5 \mathrm{~km} / \mathrm{s}\right)$, in this case the very upper layers of the star atmosphere where $\mathrm{HeI}, \mathrm{H} \beta$, and $\mathrm{H} \alpha$ form are in the contraction phase and is non-stationary.

Also if we take into account the velocity of mass center $\left(\mathrm{Vr}^{\prime}=-8.5 \mathrm{~km} / \mathrm{s}\right)$, then in phases of disappearance of $\mathrm{H} \alpha$ we observe that the deeper layers (NII, SiIII, SII, AlIII and others) of the atmosphere is almost quiet, the layers where CII and SiII form are relatively expanding. It indicates that the layers where CII and SiII form are in the relatively expansion phase, and the lower layers are in the quiet phase. Therefore we suggest that disappearance of $\mathrm{H} \alpha$ is, so to speak, the mixed conditions phase of the atmosphere. Sometimes, we observed that the $\mathrm{H} \alpha$ line shows only pure absorption component and later absorption and too small emission feature (on 2014 September 9-11). It indicates that the physical conditions in the stellar atmosphere and envelope are changeable. It is known that, the variable stellar wind in the supergiants is caused by the pulsation (Cox 1983). If the dramatic changes of the $\mathrm{H \alpha}$ profile in the spectra of $55 \mathrm{Cyg}$ are associated with the pulsation, they should occur periodically. But the amount of obtained data and their inconsistency in observation time does not make it possible to find such period in this study. On the other hand Kraus et al. (2015) noted that if the compensation of emission and absorption components of the $\mathrm{H} \alpha$ line were periodic, the possible period would be on the order of about one year.

As mentioned above, the photospheric absorption lines are variable. For instance, it was revealed that the relatively variability happens in the structures of CII $(6578.05 \AA, 6582.88 \AA)$ and HeI $5875.72 \AA$ lines, as well as in the other photospheric lines. Variability of the absorption lines indicates that disturbances are also occurring fairly deep in the atmosphere of the star. In particular and as also noted by Kraus et al. (2015), the results from the moment analysis suggest pulsations as the most reliable cause for the observed line profile variability in HeI and CII lines.

We suggest that the LPV of the photospheric lines are basically caused by non-radial pulsation (Kaufer et al., 1997).

In addition, the changes of the radial velocities and equivalent widths of lines $\mathrm{H} \beta$, HeI and CII have been revealed (see Table 2). Sometimes, the relatively large changes in radial velocity of the photospheric absorption (CII and HeI) lines are caused by the pulsation instability (Markova, \& Valchev 2000). For a more precise determination of pulsation period of star the systematic observations of this star is necessary.
Hence, observations obvious show that the $\mathrm{H} \alpha$ profile of $55 \mathrm{Cyg}$ is rapid variable. We suggest that these changes may be a manifestation of complex motions in the atmosphere of this star. Especially, the disappearance of $\mathrm{H} \alpha$ line is not accompanied by synchronous changes of the spectral parameters of the other lines formed in deeper layers of the stellar atmosphere, except $\mathrm{HeI}$ and $\mathrm{H} \beta$.

We suggest that the variations are explained probably by stellar wind properties and/or pulsations mechanisms (Kraus et al., 2015; Cox, 1983; Kaufer et al., 1997).

\section{Conclusions}

The $\mathrm{H} \alpha$ profile presented a complicated structure and a time variation for 55 Cyg star. For the first time in 2010, we observed that the profile of the $\mathrm{H} \alpha$ disappeared in the spectra. As this behaviour was observed in 2014 again we consider that this behavior is recurrent.

We suggest that in this particular case when the matter of the stellar wind moves away from the observer the absorption component of $\mathrm{H} \alpha$ is redshifted, and at that time the central frequencies of the emission and absorption components can coincide and cancel each other out, which may lead to the disappearance of $\mathrm{H} \alpha$ line profile.

We revealed that when the $\mathrm{H} \alpha$ line disappears or weaken sharply the HeI and $\mathrm{H} \beta$ lines are redshifted.

Our observations showed that the photospheric (CII, HeI etc.) line profile shapes and RVs change differently with time. We suggest that these changes in the spectra are caused by non-radial pulsations (Markova \& Puls, 2008; Kaufer et al., 1997).

\section{References}

Barlow M. J., \& Cohen D.: 1977, Astrophys. J., 213, 737. Cox J.P.: 1983, "Theory of Stellar Pulsation", Mir, Moscow (in Russian).

Crowther P. A., Lennon D. J., \& Walborn N. R.: 2006, Astron. \& Astrophys., 446, 279.

Ebbets D.: 1982, Astrophys. J., Suppl. Ser., 48, 399.

Galazutdinov G.A.: 1992, Prepr. SAO RAS, 92, 2.

Gies D. R., \& Lambert D. L.: 1992, Astrophys. J., 387, 673.

Granes P.: 1975, Astron. \& Astrophys., 45, 343.

Hutchings J.B.: 1970, Mon. Not. R. Astron. Soc., 147, 161.

Kaufer A., Stahl O, Wolf B. et al.: 1997, Astron. \& Astrophys., 320, 273.

Kraus M. et al.: 2015, Astron. \& Astrophys., 581, A75, 1.

Maharramov Y.M.: 2013, Astronomy Reports, 57, 303.

Markova N., Prinja R. K., Markov H. et al.: 2008, Astron. \& Astrophys., 487, 211.

Markova N. \& Puls J.: 2008, Astron. \& Astrophys., 478, 823.

Markova N. \& Valchev T.:2000, Astron. \& Astrophys., 363, 995.

Mikailov Kh.M., Khalilov V.M., Alekberov I.A.: 2005, Tsirk. ShAO, 109, 21.

Rosendhal J.D.: 1973, Astrophys. J., 186, 909.

Sobolev V.V.: 1947, "Moving Envelopes of Stars", Leningrad.

Sobolev V.V.: 1985, "Course in Theoretical Astrophysics", Moscow, Izdatel'stvo Nauka.

Underhill A. B.: 1960, Publ. Dom. Astrophys. Observ., 11, 353.

Wilson R.E.: 1953, Carnegie Institution of Washington D.C., Publ., 601 . 